\title{
Corela
}

Cognition, représentation, langage

HS-30 | 2020

Phonétique, littérature et enseignement du FLE : théories et recherches

\section{Enseigner/apprendre la prosodie par la lecture à voix haute de textes littéraires}

\section{Marie-Françoise Bourvon}

\section{(2) OpenEdition}

Journals

Édition électronique

URL : http://journals.openedition.org/corela/10036

DOI : 10.4000/corela.10036

ISSN : 1638-573X

Éditeur

Cercle linguistique du Centre et de l'Ouest - CerLICO

Référence électronique

Marie-Françoise Bourvon, « Enseigner/apprendre la prosodie par la lecture à voix haute de textes littéraires », Corela [En ligne], HS-30 | 2020, mis en ligne le 27 avril 2020, consulté le 22 mai 2020. URL : http://journals.openedition.org/corela/10036 ; DOI : https://doi.org/10.4000/corela.10036

Ce document a été généré automatiquement le 22 mai 2020.

\section{(c) (P)}

Corela - cognition, représentation, langage est mis à disposition selon les termes de la licence Creative Commons Attribution - Pas d'Utilisation Commerciale - Partage dans les Mêmes Conditions 4.0 International. 


\title{
Enseigner/apprendre la prosodie par la lecture à voix haute de textes littéraires
}

\author{
Marie-Françoise Bourvon
}

\section{Introduction}

1 Les enseignants le savent : la lecture à voix haute ne va pas de soi. Ils en font le constat chaque fois que les apprenants sont amenés à lire ne serait-ce que des consignes ou des énoncés d'exercices. Ce constat est conforté par l'analyse de corpus: la partie « lecture » du corpus CIL FLE ${ }^{1}$ d'un récit de 20 lignes montre que quelques minutes de préparation ne suffisent pas aux lecteurs pour produire une oralisation satisfaisante. Les résultats de l'analyse perceptive et acoustique, montrent d'une part un respect trop systématique des règles d'orthoépie, d'un respect trop automatique de la ponctuation de l'écrit lors du passage à l'oralisation et, d'autre part, l'absence de marques prosodiques susceptibles de signaler des différences de niveau d'énonciation.

2 Le laboratoire de langue permet un entraînement à la lecture ciblé sur les caractéristiques prosodiques du français (accentuation, rythme, intonation) par l'imitation d'énoncés fabriqués, le texte littéraire n'apparaissant dans les manuels de phonétique qu'en fin de leçon dans une rubrique intitulée par exemple « Lecture $^{2}$ ». La rubrique est accompagnée d'une consigne minimaliste (par exemple «lisez ${ }^{3}$ ", « lisez à voix haute ${ }^{4} »$, « déclamez $\left.[. . .]^{5} »\right)$, et lorsque (ce qui est rare) un enregistrement du texte lu est proposé, il est présenté à l'apprenant comme le moyen de vérifier sa propre lecture et non comme un modèle à imiter. C'est que lire à voix haute, surtout un texte littéraire, équivaut à interpréter et donc à faire état de sa propre compréhension. La lecture oralisée, qui demande de connaitre les «implications des formes écrites, et en particulier des signes de ponctuation, pour le rythme et l'intonation " (Conseil de l'Europe, 2001 : 92) est donc le moyen d'enseigner/apprendre certaines caractéristiques suprasegmentales du français. 
C'est pourquoi, après avoir évoqué les liens qu'entretiennent ponctuation et prosodie, et avoir rendu compte des erreurs et des manques mis en évidence dans des lectures de non natifs, nous proposerons des pistes didactiques pour un enseignement apprentissage de la prosodie en FLE par le texte littéraire. Nous montrerons comment il est possible de s'appuyer sur l'enregistrement vidéo d'une mise en scène de $L a$ Cantatrice chauve pour enseigner/apprendre à percevoir les pauses de segmentation et à les interpréter. Nous verrons aussi comment passer ensuite à la production, à partir de ce texte théâtral mais aussi d'un texte romanesque. Il s'agira alors, et la richesse polyphonique du texte littéraire s'avère ici indispensable, de s'exercer à « résoudre les équivoques » (ibid. : 92) en effectuant des choix prosodiques délibérés.

Enfin seront envisagés les bénéfices de ce type d'exercice, d'une part pour la compréhension et la lecture de textes romanesques et, d'autre part, pour l'acquisition des compétences de l'oral autres que la lecture à voix haute.

\section{Ponctuation et prosodie}

\section{1. La ponctuation du point de vue du scripteur et du point de vue du lecteur}

5 Analyser des lectures à voix haute implique de s'interroger sur la relation entre écrit et oral et, en particulier, sur la correspondance entre ponctuation et prosodie.

6 La ponctuation, qui peut être définie comme un système de signes visuels qui accompagnent le texte écrit, contribue à la production du sens. Cet ensemble, composé à la fois des signes de ponctuation, des paragraphes, des justifications et blancs graphiques, a des fonctions syntaxiques, énonciatives, sémantiques et intonatives, et se trouve en premier lieu à la disposition du scripteur. D'un scripteur dont un des objectifs est de transposer de l'oral dans l'écrit. Cependant, Blanche-Benveniste (1991:53) le rappelle : la ponctuation « ne peut pas être considérée comme une transposition exacte des phénomènes oraux d'intonation et de pauses, mais seulement comme une équivalence [...] ; elle ne peut donner que des équivalents très pauvres de l'intonation. » Catach (1991:58) va dans le même sens : pour elle le ponctème est très loin « de la parole vivante. Et pourtant, de tous les signes de l'écrit, il est le plus près de sa genèse même, "technique du corps" entre toutes, par le souffle, la respiration, la présence de l'être parlant. »

7 Valéry, cité par Meschonnic (2000), «trouvait insuffisante la ponctuation ordinaire [...] admettait faire un abus des "mots soulignés, des tirets, des guillemets" et rêvait d'intégrer à l'écriture du poème "des signes comme en musique" pour noter la vitesse ou "des arrêts de différente durée" (Cahiers 1, 1994, La Pléiade, p. 473). Des signes qui "prescrivent les modalités de l'émission" (Cahiers 2, p. 1579). Il voulait des signes de diction. »

8 Que l'on se place du point de vue de l'écriture ou du point de vue de la lecture, la ponctuation bien que d'abord au service du code écrit est donc liée à la voix, qu'elle précède l'écrit ou qu'elle en guide ou en aide l'oralisation.

9 Pour Béguelin (2002:9) «La ponctuation [...] dans la lecture à haute voix, [...] sert d'instruction pour la répartition des pauses et des modulations intonatives.» Cependant Catach (1996:98) remarque « qu'à une pause orale peut correspondre soit 
un signe équivalent, soit aucun, soit un autre signe, et inversement, qu'à un signe écrit peut correspondre soit une pause équivalente, soit aucune, soit une pause avec intonation différente. »

10 Sachant que les non natifs considèrent souvent, à tort ou à raison, que la ponctuation en français est plus aléatoire que dans leur langue maternelle (L1), on peut se demander quel usage ils font de la ponctuation lors de l'oralisation d'un texte, quel usage ils font de cette très fluctuante équivalence entre ponctuation et prosodie.

\section{2. Analyse d'un corpus CIL de lectures à voix haute}

11 L'analyse d'un corpus de lectures de non natifs donne quelques éléments de réponse. Le corpus se compose de 31 lectures enregistrées d'un texte narratif de 25 lignes: les lecteurs adultes, arabophones, anglophones, hispanophones et sinophones, sont de niveau B2 ou C1 du Cadre européen commun de référence pour les langues (CECRL). Les lectures donnent à entendre des réussites mais aussi des erreurs et celles qui reviennent le plus souvent concernent les moments où la narration fait place au discours rapporté et au commentaire.

12 L'intonation adoptée par les lecteurs lors d'un échange en forme de dialogue montre que ces passages sont identifiés comme relevant du discours rapporté, mais ce n'est le cas ni du monologue intérieur ni des commentaires. Chacun des commentaires (du type « tant pis ») est encadré par des marques de ponctuation (deux virgules ou virgule puis point), mais les lecteurs ne rendent pas toujours compte du changement d'énonciateur : en effet, les frontières droites et/ou gauches des commentaires ne sont pas marquées par des pauses de segmentation.

On sait que la pause n'est pas le seul élément susceptible de rendre compte de la ponctuation. Béguelin (2002: 10) rappelle qu'en ce qui concerne la lecture « Les normes d'orthoépie inculquées dès l'école primaire associent [...] le point à un intonème conclusif, assorti d'une pause d'une certaine durée, alors que pour une virgule, la consigne est de ne pas baisser la voix et de marquer seulement une petite pause (Damourette, 1939) ». Et ce sont bien les deux indicateurs, pause et variation intonative, que Mari Wiklund (2015) prend en compte lorsqu'elle analyse les résultats de deux tests de perception. Effectués par 31 informateurs ${ }^{6}$, ces tests demandaient, à partir des indices prosodique de la lecture des cinq premières pages de L'Étranger par Camus, de choisir, pour l'un des tests, entre point et virgule à des endroits pré-indiqués dans le texte, et pour l'autre test de les placer librement à tout endroit du texte. Les résultats montrent que lorsqu'intonation et pause coïncident, le point comme la virgule sont bien identifiés, mais que ce n'est pas le cas lorsque l'intonation, en l'absence de pause, est le seul indice prosodique. Par ailleurs, qu'il s'agisse de Campione et Veronis (2002) ou de Yoo et al. (2015) par exemple, ce sont bien la présence des pauses et leur durée qui sont prises en compte et analysées pour la synthèse de la parole. Dans notre corpus, ont donc été analysées la présence/absence et la longueur des pauses : à la fois parce que la pause apparaît comme l'hypothèse de l'indice le plus susceptible d'être perçu comme correspondant à une signe de ponctuation et parce qu'il s'agit de l'élément le plus simple à mettre en évidence en situation d'enseignement/apprentissage.

14 Après écoute de l'ensemble des lectures, les passages sélectionnés ont été analysés au moyen du logiciel Audacity à partir des fichiers .wav. Ont été mesurées en millisecondes les pauses de segmentation correspondant aux marques de ponctuation 
rendues nécessaires par l'introduction d'un «discours autre »: "points », « deuxpoints » et "virgules ». Pour que puisse être établie une comparaison entre points de fin de phrase et de fin de paragraphe, deux points de fin de paragraphe ont aussi été analysés.

Les conclusions synthétiques ${ }^{7}$ de cette analyse mettent en évidence tout d'abord, que les points de fin de paragraphe sont marqués par des pauses plus longues que les points de fin de phrase, et que tous les points (à une seule exception près et pour un seul lecteur) sont marqués par une pause, ce qui corrobore la «correspondance assez constante » signalée par Jaffré (1998). D'autre part, les deux-points, même suivis d'un blanc graphique et d'un dialogue signalé par des tirets, entraînent une pause plus courte que celle qu'entraîne un point.

Enfin, les virgules sont marquées par des pauses plus courtes que celles correspondant à des points ou à des deux-points. Par ailleurs, pour chacune des virgules analysées, une partie des lecteurs ne marque la virgule par aucune pause, et ce, qu'elle soit nécessaire ou non. Lorsque les virgules signalent l'introduction d'un commentaire, elles sont marquées par des pauses en moyenne plus longues que celles des autres virgules mesurées. Elles sont cependant perçues comme insuffisantes : l'auditeur en effet attend que la valeur polyphonique de ces passages soit marquée par des pauses qui mettent en évidence les différents niveaux d'énonciation.

Mais les lecteurs du corpus s'en tiennent trop souvent aux règles d'orthoépie, et c'est ce trop grand respect de la correspondance entre ponctuation et pause (à savoir ponctuation forte $=$ pause longue et ponctuation faible $=$ pause brève) qui est parfois source d'erreurs.

\section{3. Les pauses dans la lecture des écrivains et l'enseignement/ apprentissage de la lecture à voix haute}

18 Il n'y a bien entendu pas de relation bi-univoque entre pause et ponctuation. C'est ce que montrent par exemple les lectures que font les auteurs de leurs propres textes. Ainsi Jouhandeau adopte lors de la lecture de l'un de ses textes un rythme qui n'est pas subordonné à la ponctuation qu'il avait choisie, et Pasques $(1977: 221)$ conclut: «l'auteur, en lisant à haute voix son œuvre, est plus libre qu'un simple lecteur, il la repense, [et] en certains endroits il la recrée. »

19 Plus récemment Wilkund $(2015: 51)$ remarque que Camus, lisant les premières pages de L'Étranger, ne marque pas toujours les virgules par des pauses, qu'il marque aussi parfois le point par des traits prosodiques correspondant à une virgule et même parfois par « aucun changement prosodique ». Par ailleurs, les commentaires rapportés par Serça (2012:116) sur la façon dont Proust lisait La Recherche montrent des avis d'auditeurs pour le moins contradictoires: c'est tantôt sa "ponctuation verbale remarquable » qui est mise en avant et tantôt le fait que «son style devenait incompréhensible à l'audition ».

20 Cela implique-t-il d'ignorer la ponctuation pour l'entraînement à la lecture à voix haute ? Certainement pas si l'on se place du côté de l'enseignement/apprentissage de la prosodie en FLE. 


\section{Propositions didactiques pour l'enseignement de la prosodie par la perception et la lecture à voix haute du texte de théâtre}

\section{1. La lecture en L2: des difficultés particulières}

Alazard et al. (2009: 50) constatent « qu'à un niveau avancé, l'élocution des apprenants est beaucoup moins fluide en lecture qu'en oral spontané.» Ils s'étonnent des difficultés éprouvées par les lecteurs, alors même que ceux-ci ne sont pas « en situation de planification du discours, et [que] le phrasé est en partie indiqué par la ponctuation ", même si bien sûr, précisent-ils eux aussi, « la ponctuation ne retranscrit que très partiellement la prosodie potentielle des énoncés écrits ».

Ils déplorent par ailleurs de façon générale l'absence d'intérêt pour la prosodie en didactique du FLE et précisent aussi, concernant l'activité de lecture elle-même, que « la majorité des enseignants considèrent que si les processus de décodage sont acquis en L1, ils seront automatiquement transférés en L2. [Pourtant] l'apprenant de langue étrangère se trouve face à au moins trois tâches complexes en situation de lecture : il doit non seulement décoder la chaîne graphique et la recoder en chaîne phonémique, mais aussi organiser simultanément le matériau linguistique en groupes sémantiquement et syntaxiquement cohérents via les patrons prosodiques de la L2.» (Ibid.:50-51) Il s'agit donc prioritairement de faire prendre conscience des particularités prosodiques du français.

La première des particularités à prendre en compte concerne le possible lien entre ponctuation et pause. Pour Yoo et al. $(2015: 70)$ qui distinguent «trois niveaux différents dans la hiérarchisation prosodique » c'est "le groupe prosodique majeur » qui «correspond à une unité prosodique s'achevant par un signe de ponctuation (virgule, point, etc.), et [qui] se caractérise généralement au niveau phonétique, par la présence d'une pause.» Dans la description de Léon (2014:142), en parole spontanée, c'est le "groupe de souffle ", de longueur très variable qui se termine par une "pause audible ».

\section{2. Activités de perception dans La Cantatrice chauve}

Le premier document dont il va être question ici est la scène 1 de La Cantatrice chauve d'Eugène Ionesco. On sait que le projet de cette pièce apparaît alors qu'Ionesco tente d'apprendre l'anglais au moyen d'« un manuel de conversation franco-anglaise, à l'usage des débutants " (Ionesco, 1962:155). Pour lui, les dialogues de cette méthode ressemblent à du théâtre : il reprend alors des noms de personnages anglais et invente un étrange dialogue. Un dialogue où l'enseignant de langue retrouve quelques-uns des objectifs linguistiques d'une méthode de FLE de niveau A2: le lexique du repas, les indications de lieu, le comparatif et le superlatif entre autres, mais aussi des objectifs du niveau B, dans la répétition d'une multiplicité de connecteurs logiques. Mais la mise en scène de Lagarce ${ }^{8}$ et, en particulier, la diction adoptée par la comédienne jouant le personnage de Mme Smith constituent un support idéal pour un entraînement à la perception de phénomènes prosodiques. 
Sachant que l'objectif est ici d'interroger le lien entre ponctuation et pause, le but est d'amener les apprenants à percevoir les pauses, qu'elles soient attendues ou pas.

On commencera par demander aux apprenants (à partir du niveau B2) de marquer, dans la première réplique de Mme Smith, par une barre oblique les pauses qui correspondent à un point ou à une virgule. Il est probable que les apprenants vont segmenter ainsi le passage en percevant une pause pour chacun des signes de ponctuation :

Tiens/, il est neuf heures/. Nous avons mangé de la soupe/, du poisson/, des pommes de terre au lard/, de la salade anglaise/. Les enfants ont bu de l'eau anglaise /. Nous avons bien mangé /, ce soir /. C'est parce que nous habitons dans les environs de Londres et que notre nom est Smith.

Il est très probable que seront repérées aussi des pauses qui ne correspondent ni à un point ni à une virgule :

Tiens, il est neuf heures. Nous avons mangé de la soupe, du / poisson, des / pommes de terre au lard, de la salade anglaise. Les enfants ont bu de l'eau / anglaise. Nous avons bien mangé, ce soir. C'est parce que nous habitons dans les environs de Londres / et que notre nom est / Smith.

Dans la dernière phrase, la pause marquée entre «Londres » et « et » correspond à la syntaxe. Mais celle qui suit «notre nom est ", après un allongement très marqué de "très", met en place un suspense auquel "Smith" met un terme, soulignant ironiquement le peu d'originalité du patronyme. Il en est de même pour la pause qui sépare dans l'unité nominale « de l'eau anglaise », le nom de l'adjectif qui le qualifie ; la répétition de cet adjectif qui caractérisait déjà «la salade » fait sourire. Les pauses qui séparent respectivement «du» et "des» de "poisson» et "pommes de terre» mettent en évidence à la fois le nom des aliments (dans un contexte de présentation du lexique des aliments) et le choix du déterminant.

L'analyse de cette première réplique remet en question la concordance systématique entre ponctuation et prosodie. En effet des pauses sont introduites qui ne correspondent pas à la ponctuation, mais qui, bien entendu, induisent un sens supplémentaire. l'attention sur deux points linguistiques: les indications de lieu et la formation du comparatif.

Les pommes de terre sont très bonnes avec le lard, l'huile de la salade n'était pas rance. L'huile de l'épicier/du coin/est/de bien meilleure qualité que / l'huile / de l'épicier/d'en face, elle est même / meilleure que / l'huile de l'épicier / du bas / de la / côte. Mais je ne veux pas dire que leur huile à eux soit mauvaise.

Dans cette parodie de méthode de langue, «du coin», «d'en face» et «du bas / de la / côte» servent à distinguer trois épiciers mais servent, surtout, à présenter différentes indications de lieu, et c'est ce que met en évidence la segmentation adoptée par la comédienne. De la même façon, en marquant une pause juste avant et juste après "de bien meilleure qualité que » et "meilleure que ", c'est le comparatif qui est souligné, dans un énoncé dont la segmentation, dans un autre contexte, serait erronée.

2 La troisième réplique donne à entendre les mêmes pauses avant et après " $d u$ coin », mais introduit cette fois un superlatif :

Pourtant, c'est toujours l'huile de l'épicier / du coin / qui est / la meilleure... 

entre la deuxième et la troisième syllabe du connecteur "cependant", puis en le faisant suivre d'une pause correspondant, certes, à une virgule mais extraordinairement longue : pause « remplie» ici par 57 secondes de musique :

«Cepen / dant /, la soupe était peut-être un peu trop / salée.

Dans la septième réplique, c'est un procédé inverse qui est utilisé. D’abord, dans « [...] elle est/bonne ménagère, économe, joue du piano.", le débit rapide adopté par la comédienne et l'absence de pauses à l'endroit des virgules font de la liste des qualités que Mme Smith attribue à sa fille (qui, selon elle, lui ressemble) une sorte de vérité générale non susceptible d'être segmentée. Puis c'est l'avant-dernière phrase de la réplique qui est dite sans aucune pause :

On aurait bien fait peut-être de prendre, au dessert, un petit verre de vin de Bourgogne australien mais je n'ai pas apporté le vin à table afin de ne pas donner aux enfants une mauvaise preuve de gourmandise.

La comédienne ne marque pas les virgules encadrant " au dessert »: elle ne reprend jamais son souffle dans cette phrase (de 37 mots et de 53 syllabes) et le dernier tiers de la phrase est inaudible, preuve, s'il en est, de la fonction première des pauses qui est de respirer.

Dans ce pastiche des documents support de méthodes de langue, la diction adoptée par la comédienne permet de poser certaines bases de la prosodie du français. Les activités de repérage décrites ci-dessus permettent aux apprenants de se rendre compte que lors de l'oralisation d'un texte, ponctuation et pause peuvent correspondre, mais qu'il est aussi possible d'introduire des pauses en dehors de la ponctuation proposée (répliques 1, 2, 3, 6 par exemple), ou de ne la marquer par aucune pause (réplique 7). Ces activités permettent aussi de montrer que rapidité du débit et raréfaction des pauses vont de pair. Elles donnent à entendre la nécessité des pauses respiratoires et la fonction syntaxique des pauses grammaticales. Elles montrent aussi que les pauses d'hésitation peuvent êtres silencieuses, mais aussi non silencieuses et que si celles-ci sont alors généralement « remplies » par des « euh » ou des « um » (Duez, 1999), dans la mise en scène de Lagarce c'est par de très longues secondes de musique qu'une pause peut être « remplie».

37 Ces activités de perception permettent d'abord de signaler que le choix délibéré des pauses ajoute du sens au sens, de faire entendre ce type d'«arrêt inattendu et fortement marqué [qui est] le procédé le plus fréquemment employé par les acteurs pour mettre un terme en relief.» (Larthomas, 1995 : 68) Elles permettent aussi de faire entendre ce que la mise en scène de Lagarce et le jeu de Mireille Herbstmeyer font du texte de La Cantatrice chauve : une parodie des dialogues non authentiques des méthodes de langue.

\section{3. Activités de lecture/interprétation}

38 À la suite des activités de perception, il s'agit de proposer des activités de lecture. Il est possible tout d'abord de demander aux apprenants de préparer la lecture d'une réplique ou de répliques de la pièce :

- en faisant coïncider signes de ponctuation et pauses ;

- en introduisant des pauses non attendues et qui induisent un sens particulier; 
41

\section{西} prosodique mineur et le groupe prosodique majeur, hiérarchisation qui correspond à la distinction de Léon (2014:40-41) entre "mot phonique", "groupe rythmique" et "groupe de souffle». C'est le niveau intermédiaire, "groupe prosodique mineur » ou "groupe rythmique ", encore appelé (entre autres) "groupe accentuel» qui est ici le plus utile, car comme l'indique cette dernière dénomination, c'est celui qui est concerné par l'accentuation. L'accentuation du français n'est pas distinctive mais démarcative, et le français pour Astésano et al. $(2016: 11)$ est très probablement « la seule langue dont le domaine accentuel n'est pas le mot lexical mais le groupe de mots ». Cette particularité demande donc pour l'enseignement/apprentissage du FLE, que l'on s'y arrête. Il s'agit en effet d'amener l'apprenant à remplacer l'accent de mot caractéristique de sa L1 par l'accent de groupe du français et de lui montrer que ce ne sont ni les mots ni les blancs graphiques qui déterminent la segmentation de la chaîne parlée. Ceci conduit en particulier à signaler les phénomènes de continuité tels que les liaisons et les enchaînements consonantiques". Dans « leur huile à eux » (réplique 2) par exemple, les consonnes finales de «leur » et de "huile» s'enchaînent aux initiales vocaliques des mots qui les suivent, ce qui entraîne la resyllabation suivante : [lœ-Ruila-ø]. Concernant les liaisons, on fera remarquer la façon hyperbolique dont la comédienne signale qu'une consonne finale ne se prononce pas devant une consonne ni devant un «h dit aspiré ». Sa prononciation de "La tarte aux coings et aux haricots »

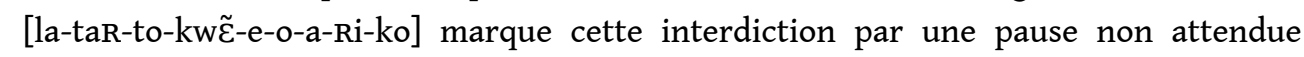
entre «aux» et «coings » ainsi qu'entre «aux» et « haricots», pause qui détruit la cohésion du syntagme nominal.

Pour Delais et al. (2008), le consensus existe sur le fait que, phonologiquement, le groupe accentuel est une séquence de syllabes dont la dernière porte un accent et que, linguistiquement, il est construit à partir d'informations syntaxiques et métriques. Le découpage en groupes accentuels peut cependant varier par exemple en raison de la variation du débit: plus le débit est rapide, moins nombreux sont les groupes accentuels, et inversement. Mais certains découpages sont impossibles, et Martin (2008) propose de considérer qu'il existe des groupes accentuables (et non plus nécessairement accentués) dans lesquels les mots sont unis par des liens de dépendance grammaticale, et que c'est un paramètre rythmique qui déterminera alors l'accentuation effective. Ces groupes comportent en moyennes 2,5 syllabes, le plus souvent trois ou quatre et très rarement plus de six syllabes (par exemple Wenk et Wioland, 1982 ; Astésano, 2001).

On l'a vu, la comédienne jouant le rôle de Mme Smith choisit de dire la fin de la réplique 7 sans y marquer de pause. On peut demander aux apprenants d'introduire des pauses en vue de les amener au découpage suivant, dans lequel les virgules encadrant « au dessert » induiraient des pauses :

On aurait bien fait peut-être / de prendre, / au dessert, / un petit verre de vin de Bourgogne australien/mais/je n'ai pas apporté le vin à table/afin de ne pas donner aux enfants / une mauvaise preuve de gourmandise.

On peut ensuite leur demander un découpage en groupes accentuels qui pourrait, par exemple, être celui-ci : 
(On aurait bien fait) (peut-être) (de prendre), (au dessert), (un petit verre de vin) (de Bourgogne australien) (mais) (je n'ai pas apporté) (le vin à table) (afin de ne pas donner) (aux enfants) (une mauvaise preuve) (de gourmandise).$$
\begin{aligned}
& \text { "de », mais qui seraient erronés dans tout autre contexte. L'objectif est bien de } \\
& \text { montrer que le découpage en groupes peut fluctuer mais que certains découpages, } \\
& \text { agrammaticaux (Delais et al., 2008), sont impossibles. }
\end{aligned}
$$

Découpage qui concernant « un petit verre de Bourgogne australien » pourrait aussi être :

(un petit verre) (de vin de Bourgogne) (australien)

ou :

(un petit) (verre de vin) (de Bourgogne australien)

ou encore :

(un petit) (verre de vin) (de Bourgogne) (australien)

Le découpage pourrait même être, pour ce qui est du début de l'énoncé, et étant donné la segmentation opérée par la comédienne dans la mise en scène de Lagarce :

*(un petit) (verre de) (vin de)

ou encore, par exemple :

*(un) (petit verre) (de) (vin) (de)

Il s'agit là de découpages qui pourraient servir à mettre en évidence la préposition "de", mais qui seraient erronés dans tout autre contexte. L'objectif est bien de agrammaticaux (Delais et al., 2008), sont impossibles.

Le texte de théâtre est fait pour être représenté et le découpage effectué ici par la comédienne met en voix un discours autre. Dans le texte romanesque, les différents niveaux de discours sont à discerner par le lecteur qui en rendra compte prosodiquement dans la lecture à voix haute.

\section{Au-delà de l'oralisation du texte de théâtre}

\section{1. Lecture d'un texte romanesque}

Le point de départ de cette réflexion était le constat des difficultés éprouvées par des lecteurs non natifs pour signaler les différents niveaux d'énonciation d'un récit. Le statut de commentaire de certains éléments du texte comme «tant pis ", par exemple, ne semble pas avoir été compris. La fin du texte, qui raconte l'arrivée très tardive de l'ami de la narratrice, est celle-ci :

Enfin j'aperçois une silhouette d'homme aux cheveux bouclés, avec un imperméable. Ça va mieux, je m'entends répondre aux excuses d'Antoine :

- Mais ça n'a aucune importance, raconte-moi plutôt comment tu vas.

Le «comment tu vas» est souvent accompagné par les lecteurs du corpus d'une intonation montante qui fait de "comment tu vas" une question directe: ce qui correspond à une tendance fréquente des lecteurs à surjouer. Dans ce passage par ailleurs, l'insuffisance de la pause après "Ça va mieux », induit un contresens : elle fait de cet élément la réponse de la narratrice aux excuses de son ami, alors que «ça va mieux " est un commentaire intérieur, et que la réponse est: "Mais ça n’a aucune importance, raconte-moi plutôt comment tu vas. » Ici, ce sont les différents niveaux d'énonciation - assez complexes il faut le reconnaître - qui, soit n'ont pas été compris, soit n'ont pas été rendus par la lecture. 
Il s'agit donc tout d'abord de montrer que «le discours littéraire est [...] le lieu d'interactions qui s'effectuent non seulement entre les personnages, mais entre le narrateur et les personnages, entre le narrateur et le lecteur " (Albert et al., $2000: 114$ ). Quelques phrases de l'incipit du Ravissement de Lol V. Stein de Marguerite Duras (1964 : 11) permettent de mettre en évidence la polyphonie énonciative du roman.

[...] Je n'ai rien entendu dire sur l'enfance de Lol V. Stein qui m'ait frappé, même par Tatiana Karl, sa meilleure amie durant leurs années de collège.

Elles dansaient toutes les deux, le jeudi, dans le préau vide. Elles ne voulaient pas sortir en rangs avec les autres, elles préféraient rester au collège. Elles, on les laissait faire, dit Tatiana, elles étaient charmantes, elles savaient mieux que les autres demander cette faveur, on la leur accordait. On danse, Tatiana? Une radio dans un immeuble voisin jouait des danses démodées - une émission-souvenir dont elles se contentaient. Les surveillantes envolées, seules dans le grand préau où ce jour-là, entre les danses, on entendait le bruit des rues, allez Tatiana, allez viens, on danse Tatiana, viens. C'est ce que je sais.

Le récit au passé, à la troisième personne, est traversé par plusieurs types de discours rapporté. Le discours direct libre au présent tout d'abord, qui n'est signalé ni par un verbe introducteur, ni par deux-points, ni par des guillemets : « On danse, Tatiana ? » et «allez Tatiana, allez viens, on danse Tatiana, viens.» : énoncés prononcés par Lol V. Stein.

Ensuite, le discours indirect libre rapporte les propos de Tatiana : « Elles, on les laissait faire, dit Tatiana, elles étaient charmantes, elles savaient mieux que les autres demander cette faveur, on la leur accordait.». Ce passage peut d'ailleurs mener le lecteur à s'interroger sur le statut du paragraphe dans son entier : récit ou discours indirect libre?

Enfin, considérons les commentaires du narrateur homodiégétique, c'est-à-dire à la fois narrateur et personnage : «Je n'ai rien entendu dire » et «C'est ce que je sais. » Ces interventions du narrateur peuvent conduire le lecteur à s'interroger sur le statut de l'énoncé entre tirets ( « - une émission-souvenir - ) : s'agit-il d'une précision apportée par Tatiana, informatrice du narrateur, ou d'un commentaire du narrateur?

Dans ces quelques lignes, on le voit, il convient tout d'abord de distinguer les différentes voix : la voix du « je », à la fois narrateur et personnage, celle de Tatiana et celle de Lol V. Stein. Il convient ensuite de déterminer comment mettre en voix ces différents niveaux de discours. Concernant l'avant-dernière phrase par exemple, une première activité demandera aux apprenants de faire coïncider ponctuation et pauses.

Les surveillantes envolées /, seules dans le grand préau où ce jour-là /, entre les danses /, on entendait le bruit des rues //, allez Tatiana //, allez viens //, on danse Tatiana //, viens.

Cela permettra de mettre au jour les statuts différents des virgules dans cette phrase : en effet, la virgule qui précède "allez Tatiana " introduit un passage de discours rapporté direct et demande donc une pause plus longue que celles demandées par les virgules précédentes. Par ailleurs, toutes les virgules de cette fin de phrase (allez Tatiana, allez viens, on danse Tatiana, viens.) demandent vraisemblablement des pauses longues, celles qu'auraient demandées des points d'exclamation (allez Tatiana! allez viens ! on danse Tatiana! viens !).

Une deuxième activité demandera un découpage en groupes accentuels qui pourrait être celui-ci : 
(Les surveillantes) (envolées), (seules) (dans le grand préau) (où ce jour-là), (entre les danses), (on entendait) (le bruit des rues), (allez Tatiana), (allez viens), (on danse Tatiana), (viens).

Ce découpage pourrait aussi être, entre autres, par exemple :

(Les surveillantes envolées), (seules dans le grand préau) (où ce jour-là), (entre les danses), (on entendait) (le bruit des rues), (allez Tatiana), (allez viens), (on danse) (Tatiana), (viens).

Mais, sous peine d'induire un contresens, il ne pourrait être :

*(Les surveillantes) (envolées, seules)

Pas plus qu'il ne pourrait être, sous peine d'être agrammatical :

*(dans le) (grand préau où ce) (jour-là)

Ces activités engageront à une réflexion sur :

la possibilité d'éliminer certaines pauses induites par la ponctuation et/ou d'en ajouter ;

la durée relative des pauses les unes par rapport aux autres ;

la possibilité d'accélération ou de décélération du débit en fonction du nombre de pauses.

Ces activités successives permettront, après une observation de « la mise en texte de la parole » (Albert et al., $2000: 181$ ) et donc une nécessaire étape de compréhension, une mise en voix des différents niveaux de discours dont les choix prosodiques seront réellement voulus.

\section{2. Vers la compréhension orale}

77 Pasdeloup (2005: 11) analyse la lecture d'un conte à débit lent, normal et rapide, et conclut qu'à un « débit lent, les variations temporelles sont sur-exploitées dans de nombreux domaines: renforcement des contrastes de durée, augmentation et allongement du nombre des pauses, ainsi qu'augmentation du nombre de schwas à l'intérieur et en fin de groupes rythmiques. » Ce qui rejoint les constats de Duez (1999) qui, analysant le rôle des pauses dans le discours politique, établit aussi un lien entre longueur des pauses et débit précisant que débit lent et pauses longues, tout comme débit rapide et pauses courtes, vont de pair. Cette corrélation, qui est perceptible dans d'autres types de discours spontané, constitue un élément de perception sur lequel s'appuyer en compréhension de l'oral. Il est possible de faire repérer les niveaux de hiérarchie prosodique dans tous les types de discours et, concernant le groupe accentuel, de faire entendre l'allongement de la dernière syllabe prononcée, très perceptible dans les énumérations par exemple. De nombreux documents donnent à entendre des accélérations et des décélérations : qu'il s'agisse de discours publicitaire ou journalistique. S'appuyer sur ces variations permet entre autres à l'enseignant de FLE de montrer que " parler vite » n'est pas une fin en soi ni l'objectif à atteindre en L2, mais correspond à des stratégies de communication.

78 C'est ce que montre par exemple l'introduction du sujet sur le salon de l'agriculture dite par Claire Chazal, sur TF1 en $2013^{10}$. Dans la transcription suivante, les pauses audibles sont indiquées par des « / "

François Hollande est donc arrivé / très tôt / ce matin / au salon de l'agriculture à

la porte de Versailles à Paris le chef de l'État / doit / passer / plusieurs heures / sur 
place / auprès des professionnels / qui n'ont/pas manqué déjà d'exprimer leurs/inquiétudes il a aussi tenu à évoquer/le scandale alimentaire de ces derniers jours / et il a fait dans ce domaine / un certain nombre de / promesses Isabelle Torre et Nicolas Belmont le suivaient

79 On attirera l'attention des apprenants sur la mise en relief de «très tôt » et de "ce matin ", et sur l'absence de pause entre : "porte de Versailles à Paris » et «Le chef de l'état ». Il est à noter que cette absence de pause concerne des éléments redondants pour un téléspectateur français, qui sait que le salon de l'agriculture se tient Porte de Versailles à Paris, et que François Hollande, en 2013, est le chef de l'état. L'information, mise en évidence par des pauses, concerne donc l'heure à laquelle François Hollande est arrivé au salon de l'agriculture et le temps qu'il y a passé. On fera aussi remarquer les pauses non attendues qui séparent déterminants et noms dans «leurs / inquiétudes » (ainsi que la liaison non enchaînée dans [lœR / zẽkjetyd]) et "un certain nombre de / promesses ». Ces pauses mettent en évidence les éléments sur lesquels la journaliste veut attirer l'attention des téléspectateurs. Le débit de Claire Chazal, qualifié de trop rapide par des apprenants de FLE, présente surtout une modification de la prosodie qui est caractéristique du style journalistique. Cette accélération du débit et la suppression des pauses concernent aussi, dans certaines publicités radiophoniques par exemple, les conditions de vente ou de crédit, c'est-à-dire les passages minimisés par le locuteur, car peu "vendeurs » et/ou censés être connus de l'auditeur. Comme l'écrivent Galazzi et Guimbretière (1987), pour développer les capacités de compréhension des apprenants, il s'agit donc à la fois de leur donner les moyens de repérer les indices prosodiques des discours et de les amener à réfléchir aux stratégies adoptées par les locuteurs.

\section{3. Vers la production orale}

On peut difficilement demander à des apprenants de s'approprier la prosodie du français par la répétition d'énoncés de conversations ou d'extraits de reportages, même si c'est de cette façon qu'Akira Mizubayashi $(2011: 85)$ raconte avoir procédé, en reproduisant grâce à ses "fréquentations radiophoniques", "les structures rythmiques [...] captées à l'écoute [...].» Des énoncés fabriqués bien ciblés permettent, au laboratoire de langue, de travailler les phénomènes de segmentation et de rythme, mais le texte littéraire montre une indéniable supériorité : les poèmes par exemple " contiennent de manière concentrée les caractéristiques rythmiques et mélodiques de la langue cible» (Dufeu, 2008:9) et, surtout, ils permettent un réinvestissement en production. Rollinat-Levasseur (2015:244-245) évoque en effet les progrès effectués par des étudiants de niveau $\mathrm{C} 1$, grâce à

la lecture de [...] textes dramatiques qui les a conduits à perfectionner leur diction en les obligeant à surarticuler les syllabes qu'ils avaient tendance à avaler à cause des caractéristiques de leur langue d'origine. [...] L'application à dire toutes les syllabes des alexandrins a donné à leur diction la régularité qu'ils n'étaient pas parvenus à lui donner jusqu'alors.

81 Ceci confirme la réalité d'un réinvestissement en parole spontanée des acquis obtenus par la lecture. Et ce en particulier pour la tendance à l'eurythmie du français : Martin (2009) la signale pour la lecture, et met le phénomène en parallèle, en parole spontanée et en lecture. Il remarque que l'eurythmie est effectuée en parole spontanée par «l'égalisation des durées successives de prononciation des groupes accentuels » et en lecture par l'«ajustement du débit de parole selon le nombre de syllabes imposé par la 
syntaxe » (Martin, 2015 : 34). Mais, en tout état de cause, le phénomène est bien présent dans les deux situations de parole. Et cette similitude montre que travailler la prosodie par la lecture de textes littéraires n'entrave en rien la spontanéité de la parole en interaction : dans les deux situations, « la fluence verbale de l'apprenant étranger, à la fois en oral spontané et en lecture oralisée, va dépendre de sa capacité à encoder la prosodie de la langue-cible » (Alazard et al., 2009 : 52).

Le corpus CIL FLE évoqué plus haut comporte une partie "entretiens »: son analyse ${ }^{11}$ montre qu'en parole spontanée aussi de nombreux locuteurs isolent les mots graphiques, et l'analyse morphologique met en évidence en particulier que des pauses apparaissent là où des déterminants, omis, étaient nécessaires. Mais l'analyse du corpus montre aussi que les structures réitératives entrainent la segmentation et l'accentuation attendues. C'est ce qui caractérise les énoncés suivants dans lesquels les groupes accentuels sont marqués par l'allongement de leur dernière syllabe prononcée. Ces énoncés sont constitués de groupes, juxtaposés ou coordonnés, construits à l'identique :

ma famille / mes frères / ma mes scurs / mon père / et ma mère /

une plage / de pierre / et de sable / les forêts / les montagnes / les paysages /

comment elle fait / comment elle parle / avec les gens /

Pour donner la possibilité à tous les apprenants d'améliorer leur prosodie, on proposera des activités de production orale qui mettent l'accent sur le lien entre prosodie et morphosyntaxe. Ces activités permettront d'attirer l'attention des apprenants sur l'importance en français des unités, qu'elles soient verbales ou nominales, et sur leur interdépendance avec la segmentation en groupes accentuels. Demander aux apprenants d'inclure dans une production orale en continu une liste (d'aliments, d'activités, de pays visités, etc.) les amène à adopter la structure rythmique $\mathrm{du}$ français. Leur demander de créer une structure réitérative dont les éléments forment des groupes de plus en plus longs, les conduit de plus à respecter le principe de progression (Lacheret, 2011) qui met en relation longueur du groupe et densité de l'information. C'est ce qu'illustre l'énoncé-authentique - suivant tiré du corpus CIL FLE :

le français / c'est romantique / c'est musical / c'est la langue de l'amour /

\section{Conclusion}

Prosodie et ponctuation «fonctionnent de façon partiellement parallèle, mais sont structurellement différent[e]s», affirme Serça (2012:70): l'enseignement/ apprentissage du FLE peut cependant s'appuyer sur ce parallélisme partiel. En effet, pour prendre conscience de la non concordance des deux systèmes, il importe pour l'apprenant de FLE d'être amené à la percevoir et d'être conduit à en comprendre les raisons. La perception des pauses, attendues ou pas, dans la diction d'une comédienne de La Cantatrice chauve, peut donc constituer la première étape d'activités permettant à l'apprenant de se rendre compte que «le locuteur marque des pauses pour respirer, pour planifier le contenu de son message, pour structurer son énoncé, pour souligner ses idées. » (Duez, 1999 : 91) Après perception et conscientisation du phénomène, puis analyse des textes, l'apprenant est capable de structurer prosodiquement des textes de théâtre ou de roman et d'en proposer une oralisation qui déjoue des ambiguïtés. 

compréhension, il sera aisé de faire entendre, par exemple, dans tout type de discours la valeur argumentative de la pause. Et la production d'un apprenant entrâné à la lecture à voix haute gardera trace des rythmes mis au jour dans les textes littéraires, puis mis en voix, qu'il s'agisse de la structuration en groupes accentuels, d'accentuation ou d'eurythmie. Il saura, à l'instar des personnages de Pour un oui ou pour un non (Sarraute, $1982: 26$ ) que placer une pause, un "suspens » entre "c'est bien » et "ça » dans « C'est bien... ça... » est tout sauf innocent.

La didactique des langues gagnerait à donner une vraie place à la lecture à voix haute. En effet, l'oralisation des textes représente - pour peu qu'elle soit formalisée - un mode de rencontre privilégié entre littérature et prosodie. Un lieu qui permet, de façon générale, de renforcer la compétence orthoépique et qui constitue l'une des très rares occasions de mise en relation étroite des deux codes de l'oral et de l'écrit.

\section{BIBLIOGRAPHIE}

Euvres littéraires

DURAS M., 1964, Le Ravissement de Lol V. Stein, Paris, Gallimard.

IONESCO E., 1954, La Cantatrice chauve, Paris, Gallimard.

IONESCO E., 1962, Notes et contre-notes, Paris, Gallimard.

MIZUBAYASHI A., 2011, Une langue venue d'ailleurs, Paris, Gallimard, coll. « Folio ».

SARRAUTE N., 1982, Pour un oui ou pour un non, Paris, Gallimard, coll. « Folio ».

Ouvrages et revues de linguistique et de didactique du FLE

ALAZARD C., ASTÉSANO C., BILLIÈRES M. et ESPESSER R., 2009, « Rôle de la prosodie dans la structuration du discours ", in H.-Y. YOO et É. DELAIS-ROUSSARIE (dir.), Actes IDP 2009 : Interfaces Discours et Prosodie (9-11 septembre 2009), p. 49-61. Disponible sur : <http://makino.linguist.jussieu.fr/idp09/docs/ IDP_actes/Abstracts/alazardetal_abt.pdf>.

ALBERT M.-C. et SOUCHON M., 2000, Les textes littéraires en classe de langue, Paris, Hachette, coll. « F / autoformation ».

ASTÉSANO C. et BERTRAND R., 2016, « Accentuation et niveaux de constituance en français : enjeux phonologiques et psycholinguistiques », Langue française, $n^{\circ} 191$, p. 11-30.

ASTÉSANO C., 2001, Rythme et accentuation en français. Invariance et variabilité stylistique, Paris, L'Harmattan, coll. « Langue et parole - Recherches en Sciences du Langage ».

ARBACH N., 2015, Constitution d'un corpus oral de FLE : enjeux théoriques et méthodologiques, thèse de doctorat soutenu à l'Université Rennes 2 sous la direction de Marie-Claude Le Bot. Disponible sur : <https://tel.archives-ouvertes.fr/tel-01147632/document>. 
BÉGUELIN M.-J., 2002, « Clause, période ou autre ? La phrase graphique et la question des niveaux d'analyse », Verbum, vol. 24, $\mathrm{n}^{\text {os }}$ 1-2, dossier : « Y a-t-il une syntaxe au-delà de la phrase » (sous la direction de M. Charolles), p. 85-107.

BLANCHE-BENVENISTE C., 1991, « Les études sur l'oral et le travail d'écriture de certains poètes contemporains », Langue française, $n^{\circ} 89$, dossier : « L'Oral dans l'écrit » (sous la direction de Daniel Luzzati), p. 52-71.

CALLAMAND M., 1973, L'intonation expressive. Exercices systématiques de prononciation, Paris, Hachette et Larousse, coll. « Le français dans le monde B.E.L.C. ».

CAMPIONE E. et VÉRONIS J., 2002, «Étude des relations entre pauses et ponctuations pour la synthèse de la parole à partir de texte ", TALN, p. 175-184. Disponible sur : <http://talnarchives.atala.org/ TALN/TALN-2002/taln-2002-long-015.pdf>.

CATACH N., 1991, «La ponctuation et l'acquisition de la langue écrite. Norme, système, stratégies », Pratiques, n 70, p. 49-59. Disponible sur : <https://www.persee.fr/doc/ prati_0338-2389_1991_num_70_1_1636>.

CATACH N., 1996 [1994], La ponctuation, Paris, PUF, coll. « Que sais-je ? ».

CONSEIL DE L'EUROPE / Division des Politiques Linguistiques (Strasbourg), 2001, Un Cadre européen commun de référence pour les langues. Apprendre, Enseigner, Évaluer (CECRL), trad. par S. Lieutaud, Paris, Didier.

DELAIS-ROUSSARIE É. et POST B., 2008, « Unités prosodiques et grammaire de l'intonation: vers une nouvelle approche », JEP-TALN 2008, Actes des XXVIII" Journées d'études sur la Parole. Disponible sur : <http://www.afcp-parole.org/doc/Archives_JEP/ 2008_XXVIIe_JEP_Avignon/PDF/avignon2008_pdf/JEP/077_jep_1565.pdf>.

DUEZ D., 1999, « La fonction symbolique des pauses dans la parole de l'homme politique », Faits de langues, $\mathrm{n}^{\circ}$ 13, dossier : Oral-Écrit : Formes et théories », p. 91-97.

DUFEU B., 2008, «L'importance de la prononciation dans l'apprentissage d'une langue étrangère », Franc-parler.org. Disponible sur : <http://www.francparler-oif.org/images/stories/ dossiers/phonetique_dufeu3.htm>.

GADET F., 1989, Le français ordinaire, Paris, Armand Colin, coll. « U ».

GALAZZI E. et GUIMBRETIÈRE É., 1987, « À l'écoute de Bernard Pivot : Stratégie de hiérarchisation des informations par la prosodie », ÉLA (Études de Linguistique Appliquée), nº66, p. 106-117.

GALAZZI E. et GUIMBRETIÈRE É., 1997, « Organisation temporelle et stratégie langagière », Travaux de didactique du français langue étrangère, $n^{\circ} 38$, p. 27-41.

JAFFRÉ J.-P., 1998, « Vers une linguistique génétique de la ponctuation », in J.-M. DEFAYS, L. ROSIER et F. TILKIN (dir.), À qui appartient la ponctuation ?, Bruxelles, Duculot, p. 243-253.

LACHERET A., 2001, « La prosodie au cœur du verbal », Rééducation Orthophonique, n² 246, p. 87-104. Disponible sur : <https://halshs.archives-ouvertes.fr/halshs-00636556/document>. LARTHOMAS P., 1995 [1980], Le langage dramatique, Paris, PUF.

LAURET B., 2007, Enseigner la prononciation du français : questions et outils, Paris, Hachette, coll. «F ».

LÉON P. R., 2014 [1992], Phonétisme et prononciations du français, Paris, Armand Colin. 
MARTIN P., 2008, «Contraintes rythmiques et syntaxiques dans la relation prosodie-syntaxe » Travaux linguistiques du CerLiCO, dossier : «Grammaire et prosodie », n² 21, p. 14-25, Rennes, PUR.

MARTIN P., 2009, Intonation du français, Paris, Armand Colin, coll. «U ».

MARTIN P., 2015, « Enjeux de la recherche prosodique pour l'enseignement du FLE » Didactique de la phonétique et phonétique en didactique du FLE, in M. BOŘEK-DOHALSKÁ et K. SUKOVÁ VYCHOPŇOVÁ (dir.), Prague, Karolinum.

MESCHONNIC H., 2014 [2000], « La ponctuation, graphie du temps et de la voix », La Licorne, n $^{\circ} 52$.

Disponible sur : <http://testjc.edel.univ-poitiers.fr/document.php?id=5856>.

PASDELOUP V., 2005, « Figures et fond dans la scène prosodique : leur résistance face aux variations du débit de parole ", Symposium Interface Discours-Prosodie-IDP05, Aix-en-Provence, France. Disponible sur : <http://www.lpl-aix.fr/ fulltext/2563.pdf>.

PASQUES L., 1977, « Ponctuation à l'écrit, arrangement rythmique à l'oral, à propos d'un conte de Marcel Jouhandeau lu par l'auteur ", in J. PETIT et N. CATACH (dir.), La ponctuation, recherches historiques et actuelles, Paris-Besançon, CNRS, p. 222-233.

ROLLINAT-LEVASSEUR È.-M., 2015, « La littérature en acte : voir, entendre, ressentir 》, in A. GODARD (dir.), La littérature dans l'enseignement du FLE, Paris, Didier, coll. « Langues \& didactique », p. 220-264.

WENK B. J. et WIOLAND F., 1982, « Is French really syllable-timed ? », Journal of phonetics, vol. 10, p. 193-216.

WILKUND M., 2015, « La perception de la ponctuation de L'Étranger d'Albert Camus à travers la prosodie de son auteur », L'Information grammaticale, $\mathrm{n}^{\circ} 145$, p. 45-52.

YOO H., DELAIS-ROUSSARIE É., LOLIVE D. et BARBOT N., 2015, « Le Rythme en Lecture Oralisée (parole synthétique et parole naturelle) », Revue française de linguistique appliquée, vol. XX, $\mathrm{n}^{\circ} 2$, p. 63-77.

\section{NOTES}

1. Corpus d'interlangue constitué par Najib Arbach, recueilli dans le cadre de l'équipe d'accueil LIDILE, EA 3874, Université Rennes 2.

2. Voir Chanèze Kamoun et Delphine Ripaud, 2016, Phonétique essentielle du français A1-A2, Paris, Didier, coll. « 100 \%FLE » et Lucile Charliac et Anne-Marie Motron, 1998, Phonétique progressive du français, Paris, CLE International, coll. « Progressive français ».

3. Chanèze Kamoun et Delphine Ripaud, 2016, Phonétique essentielle du français A1-A2, Paris, Didier, coll. « $100 \%$ FLE »

4. Dominique Abry et Marie-Laure Chalaron, 2009, Les 500 exercices de phonétique A1-A2, Paris, Hachette FLE, coll. « Les 500 exercices ».

5. Dario Pagel, Édith Madeleni, François Wioland, 2012, Le rythme du français parlé, Paris, Hachette FLE, coll. « F ».

6. 29 informateurs ayant comme L1 le finnois et langue seconde (L2) le français, mais dont les résultats ne diffèrent pas.

7. Pour le détail des résultats, voir le texte de la communication "Lire un "texte préparé" ou comment travailler la prosodie au niveau avancé », colloque du CERLICO, Linéarité et interprétation 2: Approximation, modulation, ajustement, Université Rennes 2, 11-12 juin 2015, Rennes. 
8. DVD La Cantatrice chauve, mise en scène de Jean-Luc Lagarce. Réalisation de Vincent Bataillon. Édité par [Paris] : Arte France [éd., distrib.], 2007.

9. Lorsqu'un mot se termine par une consonne prononcée et que le mot suivant commence par une voyelle, la consonne finale du premier mot devient initiale du mot suivant.

10. Extrait disponible sur : <www.encyclopediedelaparole.org>.

11. Voir Marie-Françoise Bourvon, 2014, «Prosodie et morphosyntaxe : un lien à questionner pour l'enseignement du FLE », TIPA. Travaux Interdisciplinaires sur la Parole et le Langage, $\mathrm{n}^{\circ}$ 30, dossier : «Les proéminences à l'oral» (sous la direction de Sophie Herment). Disponible sur : <http://tipa.revues.org/1084>.

\section{RÉSUMÉS}

Les enseignants considèrent vraisemblablement que la compétence de lecture à voix haute acquise en L1 est transposable en L2 : elle est donc souvent peu travaillée en cours de langue et sa place en laboratoire est réduite et non formalisée. Or la façon dont lisent les apprenants de FLE montre leur difficulté à oraliser l'écrit, en particulier lorsqu'il s'agit de marquer au moyen des pauses de segmentation la polyphonie énonciative d'un texte. Après avoir interrogé les relations entre ponctuation et prosodie, nous montrons comment certaines interprétations théâtrales mettent en évidence la valeur des pauses et comment amener les apprenants à les percevoir. Nous indiquons ensuite comment utiliser ces savoir-faire pour l'oralisation d'un texte romanesque, et posons qu'ils sont transposables en compréhension et en production orales.

Teachers apparently consider that the oral reading competence acquired in the first language can be transposed to the second language. They rarely address this issue during the language classes and when they - scarcely - do it in the language laboratory, it is in a non-formalized way. But reading aloud is definitely hard to the learners of French as a Foreign Language, particularly when they need to use segmentation pauses to show the enunciative polyphony of a text. First of all, we address the relationship between punctuation and prosody. Then, we show that theatrical interpretations can highlight the value of the pauses and explain how teachers can help learners discern them. Finally, we describe how to use those know-hows when reading a novel aloud, and state that they can be transposed to oral comprehension and production.

\section{INDEX}

Mots-clés : prosodie, texte littéraire, ponctuation, pause, lecture à voix haute.

Keywords : prosody, literary text, punctuation, pause, oral reading.

\section{AUTEUR}

MARIE-FRANÇOISE BOURVON

CIREFE - Université Rennes 2 